\title{
Detection of circulating cancer stem cell and matrix Metalloproteinase-2 in patients with hepatitis C Virus and their correlation with hepatocellular carcinoma
}

\author{
Manal Abd-Elmoneim \\ Immunology Department, Medical Research Institute, Alexandria University, Egypt
}

\section{III}

Background: Hepatitis C virus (HCV) infection is a major health problem. In majority of infected patients, HCV can effectively evade innate immunity resulting in chronic hepatitis, cirrhosis and hepatocellular carcinoma (HCC). Similar to most tumors, HCC contain poorly differentiated cancer stem cells (CSCs) that initiate tumorigenesis. The interaction of stem cell with microenvironment is key regulatory mechanism in maintenance of its self-renewal capacities. Matrix metalloproteinase (MMPs) are proteolytic enzymes that plays a role in degradation of extracellular matrix which is necessary for metastasis. Aim: The present work attempted to study the mRNA expression of CSC markers (CD133 and CD44) as well as levels of MMP-2 in HCV patients and their correlation with progression towards cirrhosis and HCC. Materials and Methods: Peripheral blood mononuclear cell (PBMC) prepared from HCV patients (either with or without complications) were probed for mRNA expression of CD133 and CD44 by RT-PCR and compared to that of non-HCV cirrhotic patients as well as healthy controls. Also, MMP-2 levels were measured using ELISA technique. Our results revealed that mRNA expression of CD133 was significantly elevated in HCV patients without complications while maximal CD44 mRNA expression and maximal circulating MMP-2 levels was noticed in HCC patients. Conclusion: These results suggests that, chronic HCV infection appear to predispose cells towards path of acquiring cancer stem cell traits by inducing CD133 and CD44 expression leads to their transformation into CSCs. In addition, HCV has important role in creating a microenvironmental change by enhancing MMP-2 release that increase invasion potential and facilitate tumor progression.

Keywords: CD133; CD44; Hepatocellular carcinoma (HCC); Hepatitis C virus (HCV); Matrix metalloproteinases-2 